\title{
An atypical presentation of frontotemporal dementia
}

\author{
William Haskins, Magdalene Y. L. Ting, Jonathan Wood, Clare Leong, Olivia Crump-Haill, Iñaki Bovill, \\ John Janssen, Chris Carswell, Ruth Mizoguchi
}

Department of Care of the Elderly Medicine, Chelsea and Westminster Hospital, London, UK

Correspondence to: William Haskins. Department of Care of the Elderly Medicine, Chelsea and Westminster Hospital, 369 Fulham Road, London SW10 9NH, UK. Email: william.haskins@doctors.org.uk.

Submitted Apr 17, 2019. Accepted for publication Sep 03, 2019.

doi: 10.21037/qims.2019.09.09

View this article at: http://dx.doi.org/10.21037/qims.2019.09.09

\section{Introduction}

Frontotemporal dementia (FTD) is a group of neurodegenerative conditions affecting the frontal and temporal lobes (1). FTD is the fourth overall most prevalent dementia subtype accounting for $<5 \%$ of cases, rising to approximately $10 \%$ in the 'early onset' (under 65) cohort (2).

FTD encompasses three overlapping clinical syndromes consisting of predominantly behavioural and executive function deficits (behavioural variant), or language deficits further categorised into semantic or non-fluent primary progressive aphasia $(1,3)$. Behavioural variant FTD (bvFTD) in the early stages typically manifests with personality change, disinhibition and social inappropriateness (1). Further, mood disturbance, compulsive behaviour and hyperphagia are also commonly observed. For this reason, adult-onset psychiatric disease can easily be misdiagnosed, not conducive to the effective management of the condition (4). Other dementia subtypes can demonstrate clinical and neuropathological overlap with FTD, although a predominance of cognitive rather than behavioural dysfunction with neuropathology extending globally beyond the frontal and temporal lobes is often observed (5).

Correct early diagnosis of FTD and mobilisation of support services is vital for optimising management, yet this is not often the case in reality. Diagnosis is multidisciplinary in nature, with collateral history, cognitive assessment and neuro imaging all integral to the diagnostic process $(1,3)$. Here, we describe a diagnostically atypical case of FTD, initially presenting following an acute hyponatraemia secondary to primary polydipsia $(\mathrm{PP})$.

\section{Case presentation}

A 66-year-old, right handed and trilingual Caucasian lady presented to hospital with a first episode of seizure. Upon admission she was found to be hyponatraemic with a plasma sodium of $108 \mathrm{mmol} / \mathrm{L}$ and had sustained fractures to $\mathrm{C} 2-$ C5 spinous processes which were managed conservatively. Her profound agitation and confusion necessitated 7 days of intensive care unit (ICU) admission with intubation to facilitate correction of hyponatraemia and enable further investigation. Following investigation (Table 1), PP was deemed causative of her hyponatraemia, and a perseverative desire to drink water persisted alongside clear cognitive impairment despite normalisation of serum sodium. Her routine blood tests were within normal ranges, excluding a vitamin B12 deficiency that was corrected. Autoimmune and infective encephalitis were excluded, and cerebral spinal fluid showed no evidence of infection. Magnetic resonance imaging (MRI) of the brain was reviewed by the neurology multidisciplinary team, including specialist neuroradiologists, who identified global cerebral atrophy with disproportionate involvement of the medial temporal, and frontal lobes; hippocampal atrophy was asymmetrical (Figure 1).

Post ICU admission, impairment of episodic memory continued, with mild fluent aphasia and compromised executive function. She also exhibited behavioural and psychological symptoms of dementia (BPSD) including agitation, aggression, wandering, and visual hallucination. She displayed hyperphagic predilection for sugary foods and 1:1 supervision was required to enforce fluid restriction; 
Table 1 Biochemical investigations of hyponatraemia

\begin{tabular}{lll}
\hline Test & \multicolumn{1}{c}{ Result } & \multicolumn{1}{c}{ Normal range } \\
\hline Serum sodium & $108 \mathrm{mEq} / \mathrm{L}$ & $135-145 \mathrm{mEq} / \mathrm{L}$ \\
Plasma osmolality & $265 \mathrm{mOsmol} / \mathrm{kg}$ & $275-295 \mathrm{mOsmol} / \mathrm{kg}$ \\
Urine osmolality & $136 \mathrm{mOsmol} / \mathrm{Kg}$ & Depends on clinical state of patient at time of sample \\
Urine sodium & $41 \mathrm{mEq} / \mathrm{L}$ & $40-220 \mathrm{mEq} / \mathrm{L}$ \\
9 am serum cortisol & $422 \mathrm{nmol} / \mathrm{L}$ & $>350 \mathrm{nmol} / \mathrm{L}$ \\
Thyroid function tests (t3, t4 and TSH) & All within normal parameters & \\
\hline
\end{tabular}

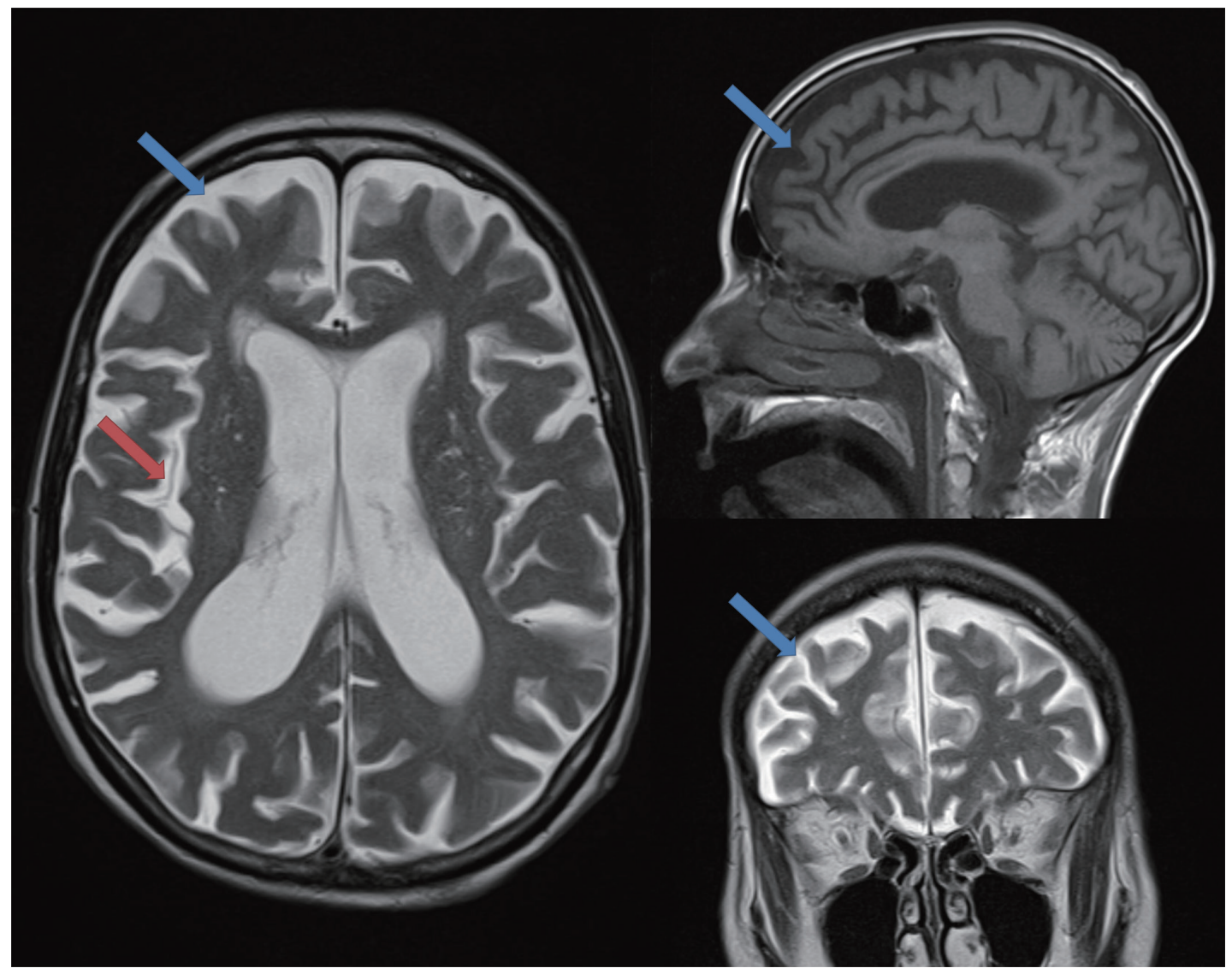

Figure 1 Axial T2 weighted (left), saggital T1 weighted (right upper) and coronal T2 weighted (right lower) views of the patient's MRI brain showed prominent frontal (blue arrows) and medial temporal lobe (red arrows) atrophy on a background of generalised cerebral atrophy. MRI, magnetic resonance imaging.

preventing relapse of water intoxication. Her original diagnosis made by a Psychiatrist was Alzheimer dementia (AD). Both donepezil and memantine were trialled but neither provided symptomatic benefit and were actually found to exacerbate symptoms.

At the multidisciplinary memory clinic, she was reviewed by a Geriatrician and Neurologist. Her family mentioned they had sought medical advice for her episodic memory 
Table 2 Neuropsychometric testing results and score interpretations

\begin{tabular}{lll}
\hline Test & Domains tested & Score achieved \\
\hline Frontal assessment & Similarities (conceptualisation) & $3 / 3$ \\
battery & Lexial fluency (mental flexibility) & $0 / 3$ \\
& Motor series & $0 / 3$ \\
& Conflicting instructions & $2 / 3$ \\
& Go-no-go (inhibitory control) & $1 / 3$ \\
& Prehension behaviour (environmental autonomy) & $3 / 3$ \\
& Total & $9 / 18[<12$ suggestive of frontal dysexecutive type dementias (6)] \\
Frontier executive & Verbal fluency & $0 / 5$ \\
screen & Inhibition & $2 / 5$ \\
& Working memory & $1 / 5$ \\
Total & $3 / 15[<7$ suggestive of frontal dysexecutive type dementias (7)] \\
assessment & Total & $20 / 30[<26$ suggestive of cognitive impairment (8)] \\
\hline
\end{tabular}

decline approximately 4 years previously, resulting in a diagnosis of anxiety and alcohol related cognitive impairment without further specialist input. Her cognitive decline accelerated 6 months prior to her hospital admission; she began wandering, and developed a compulsion to drink water, consuming up to $15 \mathrm{~L}$ per day. Neuropsychometric testing revealed severe frontal lobe dysfunction (Table 2), and she showed signs of disinhibition and repetitive compulsive behaviour. By combining the comprehensive history, neuropsychometric testing and specialist review of neuroimaging, a diagnosis of probable bvFTD was made in accordance with the international consensus criteria for bvFTD (9). Positron emission tomography (PET) scanning was considered for this patient, but in consideration of family members' wishes the team decided it would not be tolerated well and would thus not be in her best interests. Following diagnosis, memantine was stopped and trazodone was introduced which reduced her agitation.

\section{Discussion}

In humans, bvFTD is predominated by perseverative behavioural abnormalities, with hyperphagia a highly specific symptom $(1,10)$. The initially insidious decline in memory with preservation of executive function observed here was atypical for a case of bvFTD; the aberrant social function and personality change observed later on were much more emblematic of the diagnosis (10). A multidisciplinary approach utilizing a range of specialties and investigations was pivotal in obtaining the most probable diagnosis here, aided by an international diagnostic criteria; definitive diagnosis would only be achieved through post mortem histopathology or demonstration of a pathological genetic mutation (9). There is currently no standard approved pharmacological treatment for FTD. Previous pilots were suggestive of symptomatic benefit with acetylcholinesterase inhibitors as seen in $\mathrm{AD}$, but two randomised control trials failed to show efficacy $(11,12)$, a result observationally reinforced by this case.

The authors are only aware of one other published case of PP in an FTD patient (13); its aetiology remains poorly understood and potentially under diagnosed among chronic psychiatric patients (14). PP is life threatening; failure of the kidneys to excrete excess fluid results in hyponatraemia, which can lead to significant cerebral or visceral oedema and associated somatic or psychiatric symptoms termed 'water intoxication' (15). It is especially arduous and resource intensive to manage behaviourally and pharmacological strategies frequently used to manage frontal lobe symptoms such as selective serotonin reuptake inhibitors (SSRI) may exacerbate the ensuing hyponatraemia (13).

FTD remains a challenging condition to manage for 
both clinicians and caregivers. Distinct management options are available to each dementia subtype, hence early and correct diagnosis is important and enhanced by the multidisciplinary team. Awareness and vigilance for complicating features, such as PP, where possible can minimise premature deterioration of patients' condition.

\section{Conclusions}

(I) Although bvFTD typically presents with social and behavioural dysfunction, it must remain an important differential diagnosis in all patients with cognitive decline, especially those present at young onset.

(II) PP may be underdiagnosed in patients with dementia proving potentially fatal if missed.

(III) Multidisciplinary approach at memory clinics and correct interpretation of neuroimaging are vital in diagnosis of dementia to optimise both pharmacological and non-pharmacological management.

\section{Acknowledgments}

We would like to thank the patient and family discussed in this report. Written consent was obtained to publish their clinical details and clinical images.

\section{Footnote}

Conflicts of Interest: The authors have no conflicts of interest to declare.

Ethical Statement: Written informed consent for publication of their clinical details and/or clinical images was obtained from the patient and their family.

\section{References}

1. Bang J, Spina S, Miller BL. Frontotemporal dementia. Lancet 2015;386:1672-82.

2. Hogan DB, Jetté N, Fiest KM, Roberts JI, Pearson D, Smith EE, Roach P, Kirk A, Pringsheim T, Maxwell CJ. The prevalence and incidence of frontotemporal dementia: a systematic review. Can J Neurol Sci 2016;43:S96-109.

3. Warren JD, Rohrer JD, Rossor MN. Clinical review. Frontotemporal dementia. BMJ 2013;347:f4827.

4. Woolley JD, Khan BK, Murthy NK, Miller BL, Rankin KP. The diagnostic challenge of psychiatric symptoms in neurodegenerative disease: rates of and risk factors for prior psychiatric diagnosis in patients with early neurodegenerative disease. J Clin Psychiatry 2011;72:126-33.

5. Hou CE, Carlin D, Miller BL. Non-Alzheimer's disease dementias: anatomic, clinical, and molecular correlates. Can J Psychiatry 2004:49:164-71.

6. Piguet O, Hornberger M, Mioshi E, Hodges JR. Behavioural-variant frontotemporal dementia: diagnosis, clinical staging, and management. Lancet Neurol 2011;10:162-72.

7. Hutchinson AD, Mathias JL. Neuropsychological deficits in frontotemporal dementia and Alzheimer's disease: a meta-analytic review. J Neurol Neurosurg Psychiatry 2007;78:917-28.

8. Vercelletto $\mathrm{M}$, Boutoleau-Bretonnière $\mathrm{C}$, Volteau $\mathrm{C}$, Puel M, Auriacombe S, Sarazin M, Michel BF, Couratier P, Thomas-Antérion C, Verpillat P, Gabelle A, Golfier V, Cerato E, Lacomblez L; French research network on Frontotemporal dementia. Memantine in behavioral variant frontotemporal dementia: negative results. J Alzheimers Dis 2011;23:749-59.

9. Boxer AL, Knopman DS, Kaufer DI, Grossman M, Onyike C, Graf-Radford N, Mendez M, Kerwin D, Lerner A, Wu CK, Koestler M, Shapira J, Sullivan K, Klepac K, Lipowski K, Ullah J, Fields S, Kramer JH, Merrilees J, Neuhaus $\mathrm{J}$, Mesulam MM, Miller BL. Memantine in patients with frontotemporal lobar degeneration: a multicentre, randomised, double-blind, placebo-controlled trial. Lancet Neurol 2013;12:149-56.

10. Appleby BS, Tanase D. Severe hyponatremia and seizures secondary to psychogenic polydipsia in a case of frontotemporal dementia. J Neuropsychiatry Clin Neurosci 2014;26:E19-20.

11. Mercier-Guidez E, Loas G. Polydipsia and water intoxication in 353 psychiatric inpatients: an epidemiological and psychopathological study. Eur Psychiatry 2000;15:306-11.

12. Gill M, McCauley M. Psychogenic polydipsia: the result, or cause of, deteriorating psychotic symptoms? A case report of the consequences of water intoxication. Case Rep Psychiatry 2015;2015:846459.

13. Slachevsky A, Villalpando JM, Sarazin M, Hahn-Barma V, Pillon B, Dubois B. Frontal assessment battery and differential diagnosis of frontotemporal dementia and Alzheimer disease. Arch Neurol 2004;61:1104-7.

14. Leslie FV, Foxe D, Daveson N, Flannagan E, Hodges 
JR, Piguet O. FRONTIER Executive Screen: a brief executive battery to differentiate frontotemporal dementia and Alzheimer's disease. J Neurol Neurosurg Psychiatry 2016;87:831-5.

15. Nasreddine ZS, Phillips NA, Bédirian V, Charbonneau S,

Cite this article as: Haskins W, Ting MYL, Wood J, Leong C, Crump-Haill O, Bovill I, Janssen J, Carswell C, Mizoguchi R. An atypical presentation of frontotemporal dementia. Quant Imaging Med Surg 2019;9(11):1891-1895. doi: 10.21037/ qims.2019.09.09
Whitehead V, Collin I, Cummings JL, Chertkow H. The Montreal Cognitive Assessment, MoCA: a brief screening tool for mild cognitive impairment. J Am Geriatr Soc 2005;53:695-9. 